\title{
Questes
}

\section{La transmission des objets dans Guiron le Courtois (1235-1240). Affinités et exclusions}

\section{Sophie Albert}

\section{(2) OpenEdition}

\section{Journals}

\section{Édition électronique}

URL : http://journals.openedition.org/questes/629

DOI : 10.4000/questes.629

ISSN : 2109-9472

\section{Éditeur}

Les Amis de Questes

\section{Édition imprimée}

Date de publication : 15 mars 2007

Pagination : 78-88

ISSN : 2102-7188

\section{Référence électronique}

Sophie Albert, «La transmission des objets dans Guiron le Courtois (1235-1240). Affinités et exclusions », Questes [En ligne], 11 | 2007, mis en ligne le 01 janvier 2014, consulté le 22 avril 2019. URL : http:// journals.openedition.org/questes/629; DOI : 10.4000/questes.629 


\title{
La transmission des objets dans Guiron le Courtois (1235-1240) Affinités et exclusions
}

\author{
Sophie ALBERT ${ }^{1}$
}

La version de base de Guiron le Courtois, représentée par le manuscrit de Paris, BNF, fr. 350, apparaît en réalité comme la réunion de deux textes distincts $^{2}$. Le Roman de Meliadus, après un préambule consacré à Esclabor, le père de Palamède, se focalise sur 1'histoire de Meliadus de Leonois, le père de Tristan, et sur la rivalité qui l'oppose au Chevalier sans Peur, le père de Dinadan et de Brunor le Noir. En figurant les géniteurs de Palamède, de Tristan et de Dinadan, personnages majeurs du roman de Tristan en prose, l'auteur désigne d'emblée les liens de filiation, textuels et généalogiques, qui rattachent son œuvre à l'intertexte tristanien. Ces liens s'appuient sur de nombreuses allusions à la génération suivante, explicitement mentionnée par des prolepses auctoriales ou des insertions prophétiques, et évoquée, à l'arrière-plan du récit, par des échos entre les aventures des pères et des fils.

Aux antipodes de cette problématique, le Roman de Guiron, qui fait suite, dans la plupart des manuscrits, au Roman de Meliadus, semble s'ingénier à trancher toutes les attaches entre les héros, leurs fils et leurs ancêtres. Guiron, protagoniste de ce second roman, ignore tout de ses origines; son fils,

${ }^{1}$ Les pages qui suivent ont nécessairement une portée limitée ; elles seront reprises dans un article que Patrick MORAN et moi-même préparons en ce moment, et qui portera sur un corpus beaucoup plus large.

${ }^{2}$ Pour un résumé de cet ensemble, voir Roger LATHuILlÈRe, Guiron le Courtois : étude de la tradition manuscrite et analyse critique, Genève, Droz, 1966, qui présente tous les manuscrits connus en 1966. Le Roman de Meliadus correspond aux $\S 1-51$ de l'analyse, le Roman de Guiron aux § 52-130. Dans la suite du texte, les numéros de paragraphes renvoient à l'analyse de Roger Lathuillère, les numéros de feuillets au manuscrit de Paris, BNF, fr. 350. 
personnage au nom incertain, étranger d'ailleurs à la tradition arthurienne, est séparé de ses parents dès sa plus tendre enfance, et disparaît, tué par Palamède, sitôt parvenu à l’âge adulte.

Bien que ces deux romans traduisent des représentations contraires du lignage, le second brouillant systématiquement les relations de parenté alors que le premier affirme partout, et avec force, le lien héréditaire entre Meliadus et Tristan, on retrouve paradoxalement, dans un cas comme dans l'autre, un emploi similaire des transmissions d'objets, qui circulent en-dehors du cercle du genus - la parenté de sang, aussi qualifiée de charnelle, biologique ou naturelle ${ }^{3}$. De quels types de rapports ces transmissions d'objets, ainsi placées hors du champ généalogique, se font-elles le support? Modifient-elles le regard du lecteur sur la parenté naturelle et, plus largement, sur les relations unissant les acteurs du récit? Enfin, comment situer ces transmissions d'objets vis-à-vis des autres modalités possibles de la circulation des biens : don, vol, échange marchand ? Pour répondre à ces questions, j'étudierai deux passages comportant une transmission d'objets, tirés l'un du Roman de Meliadus et l'autre du Roman de Guiron.

\section{Le Roman de Meliadus : ordre du temps et formes de transmissions}

Le Roman de Meliadus prête plusieurs séjours bretons à Charlemagne, narrés au sein d'épisodes proleptiques; l'apparition de l'empereur obéit au modèle, inauguré par le Tristan en prose, des pèlerinages littéraires ${ }^{4}$. À deux

\footnotetext{
${ }^{3}$ Pour une discussion sur la pertinence et les limites de ces termes dans l'Occident médiéval, voir Anita GUERREAU-JALABERT, «Sur les structures de parenté dans l'Europe médiévale », Annales ESC, 36 (1981), p. 1028-1049 : p. 1032.

${ }^{4}$ Sur ces pèlerinages littéraires, voir Richard TRACHSLER, Clôtures du cycle arthurien. Étude et textes, Genève, Droz, 1996, p. 191-195; Disjointures, Conjointures. Étude sur l'interférence des matières narratives dans la littérature française du Moyen Âge, Tübingen, A. Francke Verlag, 2000, p. 109-111. Sur les jugements de Charlemagne dans le Roman de Meliadus, voir Barbara WAHLEN, « Nostalgies romaines : le parcours de la chevalerie dans le Roman du roi Meliadus, première partie de Guiron le Courtois », Materiali arturiani nelle
} 
occasions, l'empereur, en manière d'hommage, fait un don à une effigie célébrant la mémoire de Meliadus. Au $\S 28$, il pose une précieuse couronne sur la tête d'une statue qu'Arthur, pour la gloire du héros, a jadis érigée ; au $\S 48$, il laisse son écu et son heaume dans une chapelle dédiée à Meliadus et à Ariohan de Sessoigne. Ne pouvant analyser dans le détail ces deux épisodes, je m'en tiendrai à l'étude du second, dans lequel les transmissions d'objets entretiennent des rapports complexes avec la représentation du lien généalogique.

La scène se situe deux cents ans après la bataille qui opposa, en un long combat singulier, Meliadus à Ariohan de Sessoigne. Arthur, pour commémorer l'affrontement, a édifié une chapelle sur les portes de laquelle sont gravés les portraits des deux chevaliers. Le monument rappelle aux générations futures les héros du passé; sa fonction commémorative est illustrée par la visite de l'empereur, qui vient cautionner l'historicité des personnages romanesques en même temps qu'il salue leur vaillance ${ }^{5}$. Le sens du texte, toutefois, va plus loin que celui d'un simple procès d'autolégitimation. Entre l'empereur et les preux du passé, un lien se tisse, matérialisé par un don :

Lors entra l'empereres Charles dedens la capele et demanda ou estoit son escu, et l'en li porta, et il dist : " Por l'amor des deus chevaliers qui en ceste place se combatirent ferai ge honour en cestui leu. » Et fist adonc tout maintenant son escu pendre dedenz la chapele et son haume et tout ce leissa il leanz [...]. ( $\left.§ 48, \mathrm{f}^{\circ} 139 \mathrm{~b}\right)$

L’épisode rappelle un autre pèlerinage littéraire, raconté celui-là par la version brève du Tristan. Charlemagne ramène d'Angleterre deux reliques, les épées de Tristan et de Palamède, trouvées dans une abbaye ; après en avoir

letterature di Provenza, Spagna, Italia, Margherita LECCO (éd.), Alessandria, Edizioni dell'Orso, 2006, p. 165-181.

${ }^{5}$ Sur cette fonction de la sculpture comme «document historique », voir Colette-Anne VAN COOLPUT, «Sur quelques sculptures anthropomorphes dans les romans arthuriens en prose », Romania, 108 (1987), p. 254-267. 
évalué les mérites, il garde celle de Palamède et remet à Ogier celle de Tristan ${ }^{6}$.

Dans le Roman de Meliadus, l'empereur offre à Meliadus et à Ariohan son épée et son heaume, et au premier une couronne. Les deux romans s'accordent pour faire de l'empereur un dispensateur de trésors: dans chaque cas, il donne un bien précieux, couronne ou relique profane, à un grand personnage. Mais tandis que dans la version brève du Tristan, les épées se transmettent, très normalement, du passé au présent, elles circulent ici en sens inverse, démentant les observations de Richard Trachsler - que malgré des détours parfois incongrus, «le trajet [des objets] est toujours orienté, dans la mesure où les objets 'troyens' ou 'arthuriens' aboutissent dans la matière de France et non pas le contraire $»^{7}$. Le geste de Charlemagne, dans le Roman de Meliadus, brouille l'ordre du temps et des générations.

Les offrandes de l'empereur ne peuvent manquer d'évoquer l'un des «rôles » que lui ont fait jouer l'histoire et la fiction. Charlemagne a rapporté d'Orient de multiples reliques, distribuées ensuite à divers édifices religieux. Devenu saint, il a été au centre d'un véritable culte, dont témoignent, sur le versant germanique de l'Empire, les somptueux objets conservés longtemps à Aix-la-Chapelle et, du côté français, les légendes élaborées à l'abbaye de SaintDenis $^{8}$. La fonction médiatrice que lui confèrent les récits de transferts de reliques s'accorde avec l'image d'une émancipation vis-à-vis de l'ordre du

\footnotetext{
${ }^{6}$ Le Roman de Tristan en prose : version du manuscrit fr. 757 de la Bibliothèque nationale de France, Monique LÉONARD et Francine MORA (éds.), Philippe MÉnARD (dir.), Paris, Champion, t. IV, 2003, §331, p. 312.

${ }^{7}$ Richard TrachSLER, Disjointures..., op. cit., p. 107. L'auteur présente d'autres histoires d'épées et de heaumes (ibid., p. 99-107). Outre tous les exemples littéraires que l'on pourrait encore ajouter, les histoires de reliques de "saints guerriers » récupérées, voire disputées après la mort du saint par des seigneurs, existent aussi dans la réalité médiévale. Voir par exemple à ce sujet l'article de Michel LAUWERS, «À propos de l'usage seigneurial des reliques : note sur les 'Miracles de Saint Gengoul' (1034 ou 1045)», Guerriers et moines. Conversion et sainteté aristocratiques dans l'Occident médiéval (IX ${ }^{e}-X I I^{e}$ siècle), Michel LAUWERS (éd.), Antibes, APDCA, 2002, p. 285-288.

${ }^{8}$ Voir sur ce point Edina BozóKY, La Politique des reliques, de Constantin à Saint Louis, Paris, Beauchesne, 2006, p. 135-140 et 195-199.
} 
temps : doué d'une «singulière puissance de conciliation $»^{9}$, l'empereur abolit les distances spatiales et temporelles.

Or, l'ordre naturel du temps est rétabli, d'une certaine manière, par le biais d'un autre mode de transmission, figuré conjointement à l'hommage post mortem. Le $\S 48$ met en scène, aux côtés de Charles, Ogier de Danemark. Après avoir loué les mérites de Meliadus, l'empereur désigne à son comparse «l'ymage »d'Ariohan :

Celui fu vostre parent et li droit encomencement de vostre lygnage. Et certes, se vos fuissiez si prodome d'armes com il fu, assez vos porriez prisier; et de ce solement que vos fustes estrait de sa generacyon et de son sanc vos devez vos plus chier tenir et en devriez trop mielz valoir. $\left(\S 48, \mathrm{f}^{\circ} 139 b\right)$

Le lien de parenté entre les deux guerriers est explicité quelques lignes plus haut. Une fois vaincu par Meliadus, Ariohan part pour le Danemark. Devant « sa bone chevalerie », les Danois prient le roi de donner au guerrier « sa fille por moillier», et le souverain s'exécute. Ariohan épouse la jeune femme et, à la mort du roi, monte à son tour sur le trône ; « et de cels qui issirent puis de lui par droit legnaige fu estrait Ogyers le Danois » (§48, $\left.\mathrm{f}^{\circ} 138 \mathrm{~d}\right)$. Chevalier admirable, ayant par sa vertu gagné la main et le royaume d'une princesse étrangère, Ariohan réunit toutes les qualités du bon chef de lignage ${ }^{10}$. Personnage décalé par rapport à la matière de Bretagne, il est épargné par la fin du monde arthurien : la continuité généalogique entre le guerrier saxon et Ogier

${ }^{9}$ Je reprends la formule à Jean-Pierre ALBERT, Odeurs de sainteté. La mythologie chrétienne des aromates, Paris, Éditions de l'EHESS, 1990, p. 273, qui l'applique à sainte Hélène.

${ }^{10}$ Sur ce mode de construction généalogique, voir Georges DUBY, «Structures de parenté et noblesse dans la France du Nord aux $\mathrm{XI}^{\mathrm{e}}$ et $\mathrm{XII}^{\mathrm{e}}$ siècles » et "Remarques sur la littérature généalogique en France aux $\mathrm{XI}^{\mathrm{e}}$ et $\mathrm{XII}^{\mathrm{e}}$ siècles ", La Société chevaleresque. Hommes et structures $d u$ Moyen Âge, Paris, Flammarion, 1988 (première éd.: Paris, Éditions de l'EHESS, 1979), p. 143-166 et 167-180. 
le Danois n'est pas affectée par la rupture que représente, pour beaucoup de lignées, ce « crépuscule de la chevalerie » qu'est la bataille de Salesbières ${ }^{11}$.

La présence d'Ogier aux côtés de l'empereur induit un parallélisme formel entre les «visiteurs » et les ancêtres : aux deux statues d'Ariohan et de Meliadus répond, en quelque sorte, le couple que constituent Ogier et Charlemagne. Mais tandis qu'Ogier descend directement d'Ariohan de Sessoigne, Charlemagne n'est nullement apparenté à Meliadus. À chaque guerrier carolingien est associée une relation particulière au chevalier défunt: d'un côté, un lien généalogique ; de l'autre, une affinité élective, exprimée par la transmission d'objets emblématiques. Chaque type de relation engendre un rapport spécifique à la temporalité : alors que la parenté biologique est soumise aux contraintes de la successivité temporelle, l'élection de Meliadus par Charlemagne, et le don d'objets destiné à la symboliser, en sont totalement affranchis ${ }^{12}$. Les liens du sang manifestent l'appartenance à un même genus aristocratique ; ils relèvent de «l'immanence aristocratique et féodale $»^{13}$ et se situent, si l'on peut dire, du côté de la chair. Le lien électif qui unit, par delà les siècles, Charles et Meliadus, fondé sur l'adhésion à des valeurs communes, procède d'une forme de parenté artificielle.

${ }^{11}$ Selon le titre du recueil La Mort du roi Arthur ou Le crépuscule de la chevalerie, Jean Dufournet (éd.), Paris, Champion, 1994.

${ }^{12}$ Jérôme BASCHET a théorisé ce type de dénégation des règles de la parenté charnelle, dans le registre de la parenté divine; il parle d'anti-généalogie (Le Sein du père. Abraham et la paternité dans l'Occident médiéval, Paris, Gallimard, 2000, «La parenté divine », p. 29-62 : p. 58). Dominique Donadieu, lors d'une conférence sur les «Figurations de la parenté spirituelle : le cas des ordres religieux », a apporté une nouvelle preuve de la fécondité du concept de Jérôme BASCHET. Parmi les images qu'elle a commentées, plusieurs donnaient une formule iconographique à l'émancipation de la structure des ordres vis-à-vis du cours naturel du temps (conférence donnée le 6 décembre 2006 dans le cadre du séminaire de l'EHESS «Famille, pouvoir et religion », organisé par Philippe MAURICE et Abel LAMAUVINIĖRE).

13 Dominique BouteT, «Royauté et transcendance dans la fiction littéraire au temps de Philippe Auguste », Personne, personnage et transcendance aux XII et XIII siècles, MarieÉtiennette BÉLY et Jean-René VALETTE (éds.), Lyon, Presses Universitaires de Lyon, 1999, p. $35-59$ : p. 39. 
Sans aller jusqu'à affirmer, selon les mots d'Anita Guerreau-Jalabert, qu'«associée à la sexualité, au péché originel, au diabolique, la parenté charnelle (consanguinité et alliance) est automatiquement disqualifiée comme modèle de relations sociales $\rangle^{14}$, force est de constater qu'Ogier et Ariohan, face à Meliadus et à Charlemagne, font figure de parents pauvres. Les deux Saxons ne sont pas les seuls à pâtir de la comparaison avec cette affinité élective ; l'autre victime, c'est Tristan, ce Tristan qui n'est jamais que le fils de son père... En illustrant le lien privilégié de l'empereur et du héros, le Roman de Meliadus relègue l'amant de Cornouaille à un mode moins glorieux d'inscription dans le monde.

Cette relative dévalorisation trouve un écho dans le jugement émis par l'empereur au début du passage. Au rebours des incidentes du narrateur ou des relais de la voix merlinienne, qui présentent Tristan, selon les termes de Gilles de Rome, comme la «perfection» de Meliadus ${ }^{15}$, Charlemagne, en vertu d'un système de valeurs qui place la prouesse guerrière devant l'amour courtois, inverse la hiérarchie entre le père et son enfant :

Ge diroie que li rois Melyadus valut mielz que Tristan, et vos dirai adonc rayson por quoi. Ce que Tristan fist, selonc ce que ge voi, il fist auques por amor et les tres grant fait qu'il fist, il n'eust fait, se ne fust por amor qui de ce fait le contretenoit et qui mainte fois le mist en mortel perill, por quoi ge di qu'il y fist plus par force d'amor que par force de son cors. Amor l'estoit aguillon et esperon qui de bien faire le contenoit. Ensint com ge vos cont estoit de Tristan. Mes del roi Melyadus ne puis ge mie ce dire, se ge m'en voill aler par la droite verité, car tot ce qu'il fist, il ne fist mie par force d'amor, mes par force de son cors tant solement; de son propre bonté li vint a faire bien et non mie par force d'amor. Et ne pour quant, ge di bien que Tristan fist plus assez que ne fist mie sis peres. $\left(\S 48, f^{\circ} 139 a\right)$

14 Anita GUerreau-JALABert, «Nutritus / oblatus: parenté et circulation d'enfants au Moyen Âge », Adoption et fosterage, Mireille CorbIER (éd.), Paris, De Boccard, 1999, p. 263-290 : p. 285. L'auteur oppose aux liens du sang l'idéale fraternité que crée le fosterage.

${ }^{15}$ Cité par Didier LETT, «'L'expression du visage paternel'. La ressemblance entre le père et le fils à la fin du Moyen Âge : un mode d'appropriation symbolique », Cahiers de Recherches Médiévales (XIII ${ }^{e}-X V^{e}$ siècles), Être père à la fin du Moyen Âge, 4 (1997), p. 115-125 : p. 116. 
Le message est clair : bien que Tristan - tradition littéraire oblige ! - ne laisse pas d'être un plus grand héros que Meliadus, le père, pour avoir su prouver sa valeur intrinsèque sans recourir à la «force d'amor », mérite plus d'éloges que le fils. Il s'agit de créer une communauté idéologique entre Meliadus et Charlemagne, et d'en exclure l'amant de Cornouaille.

En montrant, à travers la transmission de différents objets, les affinités électives entre Meliadus et Charlemagne, le Roman de Meliadus met en scène un lien concurrentiel à la parenté naturelle : à Tristan l'amoureux fait pendant le guerrier épique, qui reconnaît dans Meliadus un idéal compagnon d'armes. La juxtaposition des deux modèles de transmission, «spirituel» et généalogique, instaure une tension, hautement conflictuelle, entre deux systèmes de valeurs.

La question, dans le Roman de Guiron, se pose en d'autres termes; elle est, d'une certaine manière, beaucoup plus simple, dans la mesure où la parenté naturelle est tout bonnement évincée. Néanmoins, les transmissions d'objets y revêtent également deux fonctions complémentaires : elles relient en même temps qu'elles disjoignent, elles disent tout à la fois l'affinité et l'exclusion.

\section{L'épée d'Hector le Brun dans le Roman de Guiron}

La première séquence du Roman de Guiron raconte, entre autres aventures, comment le héros se laisse séduire par la dame de Malehaut, mariée à son meilleur ami. À la suite de circonstances qu'il serait superflu de résumer, Guiron et la dame se retrouvent ensemble, et seuls, au beau milieu de la forêt ; ils s'engagent dans un sentier détourné, arrivent auprès d'une fontaine et descendent de leur monture. Le chevalier

oste son hiaume et met son escu d'une part et son glaive ; et s'espee met devant lui droitement desus la fontainne. Cele espee ot maint jour portee li bons chevaliers, li vaillant, que l'en apeloit Hector le Brun. Et pour 
l'amor de lui et pour ce que l'espee estoit trop bonne de soi, l'amoit Guron plus chierement que ne faisoit li rois Artus le meillour chaustel qu'il avoit. $\left(\S 65, \mathrm{f}^{\circ} 167 \mathrm{c}\right)$

Alors que les deux personnages s'apprêtent à accomplir leur «vilain talant $»\left(\S 65, \mathrm{f}^{\circ} 167 \mathrm{c}\right)$, la lance du chevalier tombe sur son épée, qui tombe dans la fontaine. Guiron accourt pour ramasser son arme.

Si l'en relieve et la trait del fuerre et la commence a essuer au pan de sa chemise. Et quant ce vient a essuer le pomel de l'espee, il commencha a regarder letres qui la estoient escrites; et ces letres furent entaillies par occoison del bon chevalier qui Ector le Brun estoit apelés. Et les letres qui illuec estoient entaillies disoient ces propres paroles : «Loiauté passe tout et trahison honnist tout homes dedens cui ele se herberge $»^{16}$.

Désespéré d'avoir manqué aux devoirs de l'amitié et d'avoir démérité visà-vis d'Hector, Guiron décide de prendre «haute vengance » de lui-même ; après une ode à la valeur de son épée et à son premier possesseur, il se frappe «si roidement qu'il s'en tresperce ansdeus le quisses ${ }^{17}$. La dame, se précipitant sur lui, le retient d'achever son office.

L'épisode apparaît comme une réécriture d'un passage de la Queste del Saint Graal dans lequel Perceval, emporté sur une île déserte, doit faire face aux assauts de la tentation. Le deuxième jour, vers midi, il reçoit la visite d'une trop belle demoiselle. Comme il est sur le point de succomber au péché de luxure, il s'aperçoit que son épée est négligemment jetée à terre ; lorsqu'il se penche pour la redresser, il est arrêté par une croix vermeille gravée sur le pommeau de l'arme ${ }^{18}$. Reprenant soudain ses esprits, il se signe, tandis que la demoiselle s'éloigne dans une puanteur proprement infernale. Perceval, pour payer à Dieu

${ }^{16}$ Les deux passages sont cités d'après "Guiron le Courtois » : une anthologie, Richard TrACHSLER (dir.), Alessandria, Edizioni dell'Orso, 2004, p. 196. Dans cet ouvrage figure l'ensemble du passage, depuis le moment où Guiron et la dame se retrouvent en tête à tête jusqu'au suicide manqué du héros.

${ }_{17}^{1}$ Ibid., p. 200 pour les deux citations.

${ }^{18}$ La Queste del Saint Graal, roman du XIII siècle, Albert PAUPHILET (éd.), Paris, Champion, 2003 (première éd. : 1923), p. 104-115. 
son « amende », s'inflige une blessure à la « senestre cuisse ». Plus tard, un saint homme viendra lui révéler, en une longue glose, le sens de l'aventure: la demoiselle était, on l'aura deviné, l'incarnation du diable.

De la Queste au Roman de Guiron, les coïncidences sont frappantes : même abandon du héros convié au péché de luxure, même mouvement vers une épée tombée trop bas et qu'il faut redresser, même prise de conscience, subite et salvatrice, grâce à un signe «entaillé » dans la poignée de l'arme. Dans la « haute vengance » que Guiron déclare perpétrer comme dans 1'《amende » offerte à Dieu par Perceval, un même champ sémantique s'exprime, celui de la réparation de l'offense ; en se frappant la « cuisse », désignation euphémisée des organes sexuels, le héros stigmatise le membre par lequel il aurait pu pécher.

Ces similitudes, cependant, ne doivent pas masquer les déplacements qu'introduit le Roman de Guiron. D'une part, le texte oppose un silence absolu à la longue élucidation qui suit, dans la Queste, l'aventure de Perceval. Aucun indice ne fait signe vers une quelconque transcendance, aucun ermite ne vient expliquer à Guiron la «senefiance » de ses actes. Parallèlement, en même temps que la portée chrétienne de l'aventure s'efface, une éthique chevaleresque se fait jour, qui exalte, au lieu de la foi, l'amitié virile ; la transposition axiologique est manifeste, du registre célestiel au registre profane. Enfin, le Roman de Guiron ajoute à son intertexte un motif, celui, précisément, de la transmission de l'arme : Guiron possède l'épée d'Hector le Brun, cité dans l'ensemble du roman comme un modèle insurpassable. Ce personnage, mort au moment du récit principal, n'est présenté nulle part comme le parent ou l'ancêtre de Guiron le Courtois $^{19}$. Entre les deux héros, on observe en revanche des jeux d'analogie, chacun étant confronté, en une occasion au moins, à une situation où il doit choisir entre l'appel d'une séduction féminine et la fidélité à l'amitié entre

\footnotetext{
${ }^{19}$ De ce point de vue, la critique fait une confusion : s'appuyant sur les réfections tardives du roman, où Guiron devient le parent d'Hector le Brun et du fils de ce dernier, Galehaut le Brun, elle parle du «lignage des Bruns» pour désigner le lignage du héros. Dans les manuscrits du XIII ${ }^{\mathrm{e}}$ siècle, Guiron n'est pas apparenté aux deux Bruns.
} 
hommes $^{20}$. Mais si Hector le Brun fait passer sans hésitation l'ami devant la femme, Guiron, dans l'épisode analysé, n'est rappelé à ses devoirs que grâce à l'inscription gravée sur son épée, une épée qu'Hector, justement, lui a léguée.

La transmission de l'arme établit ainsi entre Hector et Guiron une relation morale, l'épée se faisant le symbole d'une valeur à préserver; elle s'accompagne en même temps d'une dégradation, Guiron demeurant en deçà de son prédécesseur. L'ensemble est empreint d'une misogynie tout à fait remarquable, et le motif de la transmission, dans ce contexte, est employé aussi bien pour exprimer le lien qui unit le donateur au donataire que pour exclure de ce lien la féminine engeance.

J'ai laissé de côté un détail, qui n'est pourtant pas anodin. Alors que Guiron gît à terre, rougissant l'herbe de son sang, un chevalier survient, qui a assisté à la scène. Il essaie par deux fois de dérober à Guiron son épée, et le héros, par deux fois, se relève et l'effraie, l'empêchant de lui prendre l'arme.

L'incident met au jour deux formes antagonistes de circulation des biens, le vol et la transmission. Par son déroulement, il éveille le souvenir de la mort de Roland. Comme le neveu de Charlemagne se pâme sur l'herbe tendre, un Sarrasin s'approche et se saisit de Durendal ; Roland, sentant qu'on lui prend son épée, tue le voleur d'un coup de cor. Or, Durendal, dont le héros, en des laisses célèbres, magnifie la vertu, a une histoire peu commune :

Carles esteit es vals de Morïane,

Quant Deus del cel li mandat par sun angle

Qu'il te dunast a un cunte cataignie ;

Dunc la me ceinst li gentilz reis, li magnes ${ }^{21}$.

\footnotetext{
${ }^{20} \mathrm{Au} \S 65$ pour Guiron (c'est l'épisode analysé), au $\S 78$ pour Hector le Brun. Je me permets de renvoyer sur ce point à mon article «Échos des gloires et des 'hontes'. À propos de quelques récits enchâssés de Guiron le Courtois (ms. de Paris, fr. 350) ", à paraître dans la Romania, 124 ( $1^{\mathrm{er}}$ semestre 2007).

${ }^{21}$ La Chanson de Roland, Ian SHORT (éd.), Paris, Librairie Générale Française, 1990, laisse 172, p. 164.
} 
La transmission de l'épée fait intervenir deux personnages doués d'une fonction médiatrice: l'ange, messager entre ciel et terre, et l'empereur, destinataire privilégié, dans la Chanson de Roland, des manifestations divines. L'origine de cette transmission réside dans la grâce de Dieu, c'est-à-dire dans un don gratuit, opposé en cela à l'échange commercial. Le vol, à l'inverse, est le fait d'un Sarrasin; s'il ne ressortit pas davantage à une logique marchande, il vaut surtout, dans cet exemple, comme le contraire du don ${ }^{22}$.

On retrouve ces distinctions dans le Roman de Guiron, mais dépourvues, conformément à la tonalité profane du roman, de tout enjeu spirituel. C'est un chevalier, et non un émissaire divin, qui a donné à Guiron son épée ; et c'est un autre chevalier, aucunement décrit comme un païen, qui s'efforce en vain de lui voler son arme. La ligne de partage entre la transmission et le vol et, corollairement, entre le bon chevalier et le traître, n'en est pas moins très clairement tracée.

Dans le Roman de Meliadus comme dans le Roman de Guiron, les transmissions d'objets soutiennent des affinités électives au regard desquelles les autres modes de relations paraissent dépréciés. Ceux-ci relèvent dans le Roman de Guiron de l'amour adultère, et dans le Roman de Meliadus de la parenté naturelle, incarnée par la filiation entre Meliadus et Tristan et par le lien généalogique qui rattache Ogier à Ariohan. Dans les deux cas, le statut de la chair est en jeu, chair coupable ou chair innocente, toujours dévalorisée par

22 Mon attention a été attirée sur cet aspect par l'article de Philippe HaUgEARD, «Le magicien voleur et le roi marchand : essai sur le don dans Renaut de Montauban ", Romania, 123 (2006), p. 292-320. L'auteur met en évidence l'opposition et la complémentarité entre le vol et le don, et montre que quand le don est grippé, le seul recours réside dans le vol ; ainsi s'explique la possibilité d'une fonction positive pour le vol. Le Roman de Guiron présente une configuration inverse, et, pourrait-on dire, «normale » : quand le don fonctionne bien, le vol est marqué négativement. Dans un domaine très différent, Patrick J. GEARY a montré que le vol faisait partie des procédés légitimes d'acquisition des reliques, dans Le Vol des reliques au Moyen Âge : furta sacra, Paris, Aubier, 1993 (première éd. : Princeton, 1978 ; deuxième éd. revue et augmentée : Princeton, 1990). 
rapport aux liens idéaux concrétisés par les transmissions d'armes. Aussi ces transmissions combinent-elles deux fonctions en apparence contradictoires, et en réalité logiquement articulées : tout en tissant des liens entre les chevaliers, et justement parce qu'elles contribuent à rendre ces liens suffisamment visibles, elles dessinent les contours d'un espace dont d'autres sont exclus.

On pourrait se demander ce qui motive le discrédit dont sont frappées les relations charnelles. Que l'amour adultère puisse être critiqué, on ne saurait s'en étonner ; mais que cette suspicion s'étende au lien filial, à la parenté biologique, pourtant valorisés dans la société médiévale, ne laisse pas de surprendre. Plusieurs explications peuvent être avancées. D'une part, comme l'a montré Philippe Haugeard, la littérature du XII ${ }^{\mathrm{e}}$ siècle fait largement écho aux problèmes d'héritage que rencontrent les fils de l'aristocratie; dans l'espace de la fiction, les chevaliers frustrés trouvent souvent au gré de l'aventure un père de substitution, apte à les fournir largement en femmes et en fiefs ${ }^{23}$. Si ces questions ne se posent plus, ou se posent différemment, dans les romans en prose, elles ont pu infléchir l'image de la paternité dans un sens négatif, ou conduire les écrivains à inventer, à côté de la famille biologique, des parentés parallèles pourvues de vertus éminentes. La fraternité rituelle unissant les compagnons de la Table Ronde, ou le fait qu'Arthur apparaisse comme l'adoubeur par excellence des chevaliers errants, offrent à cette hypothèse des arguments probants. D’autre part, l'Église médiévale a institué une hiérarchie entre la parenté charnelle et la parenté spirituelle, en accordant la primauté à la seconde; on peut désormais affirmer, grâce aux analyses d'Anita GuerreauJalabert $^{24}$, que cette hiérarchie est transposée dans la littérature, et réinterprétée à la lumière des valeurs chevaleresques. Dans cette perspective, la transmission

${ }^{23}$ Philippe Haugeard, Du «Roman de Thèbes » à "Renaut de Montauban » : une genèse sociale des représentations familiales, Paris, PUF, 2002. Voir surtout la troisième partie, «L'héritage, le père et les fils : constructions imaginaires ». Dans ce bulletin, l'article de Vanessa OBRY aboutit à des conclusions similaires.

${ }^{24}$ Voir notamment, outre les articles déjà cités, « Aliments symboliques et symbolique de la table dans les romans arthuriens (XII ${ }^{\mathrm{e}}$-XIII ${ }^{\mathrm{e}}$ siècles) », Annales ESC, 47 (1992), p. 561-594. 
d'un objet met en exergue, au sein du domaine romanesque, un type de relation profane conçu sur le modèle de la parenté spirituelle.

Reste encore à comprendre pourquoi les écrivains ont choisi, pour servir de support à ces liens électifs, des transmissions d'armes ou de couronnes. La réponse, me semble-t-il, réside dans la manière même dont ces objets circulent. Les transmissions d'objets de Guiron le Courtois procèdent d'un don gratuit, d'autant moins contraint qu'il se fait en dehors des règles d'héritage qu'auraient $\mathrm{pu}$ induire des liens de filiation, et d'autant moins intéressé qu'il est à sens unique : aucun contre-don ne vient payer le donateur de l'objet dont il se départ, aucune rétribution marchande n'en finance le coût. On pourrait, en termes chrétiens, évoquer la circulation de la grâce ou de la caritas; on pourrait aussi évoquer l'idéologie de la largesse royale, décalée, dans le cas du Roman de Guiron, sur l'ensemble de la classe chevaleresque - ou du moins des «bons » chevaliers $^{25}$. Par sa mobilité, l'objet se prête tout particulièrement à des déplacements, concrets et métaphoriques; il dit la disjonction entre des liens charnels et des liens électifs, idéalisés par la mise en pratique d'un don purement gracieux.

25 Ce décalage s'observe également dans la pratique de la vengeance: le pardon, manifestation de la grâce divine, est le fait des chevaliers eux-mêmes, qui n'ont besoin ni des clercs ni du roi pour régler leurs litiges. Dans le Roman de Guiron, ce constat se vérifie d'autant mieux que le roi Arthur n'apparaît jamais dans l'action principale : pas d'Arthur, pas de justice ni de pardon royaux. Ce vide laisse toute la place aux dons (et aux pardons) chevaleresques. 